\title{
On-site manipulation of single whole-genome DNA molecules using optical tweezers
}

$\operatorname{AUTHOR}(\mathrm{S}):$

Oana, H; Kubo, K; Yoshikawa, K; Atomi, H; Imanaka, $\mathrm{T}$

\section{CITATION:}

Oana, H ... [et al]. On-site manipulation of single whole-genome DNA molecules using optical tweezers. APPLIED PHYSICS LETTERS 2004, 85(21): 5090-5092

\section{ISSUE DATE:}

2004-11-22

URL:

http://hdl.handle.net/2433/39665

\section{RIGHT:}

Copyright 2004 American Institute of Physics. This article may be downloaded for personal use only. Any other use requires prior permission of the author and the American Institute of Physics. 


\title{
On-site manipulation of single whole-genome DNA molecules using optical tweezers
}

\author{
Hidehiro Oana ${ }^{\text {a) }}$ \\ Department of Mechanical Engineering, The University of Tokyo, Tokyo 113-8656, Japan \\ Koji Kubo and Kenichi Yoshikawa \\ Department of Physics, Kyoto University and CREST, Kyoto 606-8502, Japan \\ Haruyuki Atomi and Tadayuki Imanaka \\ Department of Synthetic Chemistry and Biological Chemistry, Kyoto University, Kyoto 615-8510, Japan
}

(Received 12 May 2004; accepted 22 September 2004)

\begin{abstract}
In this letter, we describe a noninvasive methodology for manipulating single Mb-size whole-genome DNA molecules. Cells were subjected to osmotic shock and the genome DNA released from the burst cells was transferred to a region of higher salt concentration using optical tweezers. The transferred genome DNA exhibits a conformational transition from a compact state into an elongated state, accompanied by the change in its environment. The applicability of optical tweezers to the on-site manipulation of giant genome DNA is suggested, i.e., lab-on-a-plate. (C) 2004 American Institute of Physics. [DOI: 10.1063/1.1825064]
\end{abstract}

Over the past decade, great advances have been made in our understanding of the base-sequence in genomic DNA, including the human genome. ${ }^{1}$ The full length of genomic DNA is rather large; on the order of millimeters to centimeters. The separation and characterization of giant genomic DNA is still a matter in which tremendous labor is necessary. Giant DNA molecules are fragile to physical treatment such as pipetting and usually analyzed after fragmentation into small pieces by use of restriction enzyme. After the procedure of the fragmentation, information on the physical distance between different genes is completely lost. Invention of a nondestructive method on giant DNA is awaited for the further development of biological science. Here, we will show that optical tweezers ${ }^{2}$ are applicable for the nondestructive, on-site handling of individual $\mathrm{Mb}$-sized genomic DNA, using Thermococcus kodakaraensis (KOD1). ${ }^{3}$ KOD1 has a single 2088 737-bp DNA $(\sim 700 \mu \mathrm{m})$ as the whole genome in the cell body, ${ }^{4}$ and is an interesting organism because it can maintain its life with such a minute amount of DNA. The genome DNA is highly folded and exists in the cell by forming a complex with cationic histone-like proteins. ${ }^{5}$ High salt conditions lead to the dissociation of nucleoproteins from DNA and consequently change the higher-order structure of DNA. ${ }^{6-8}$ Using this characteristic, we tried to recover an intact single whole-genome DNA and change its higher order structure by controlling the surrounding buffer conditions under a microscope by means of optical tweezers. All of these procedures were successfully performed in a small confined space, i.e., within a sandwich of cover slips.

KOD1 cells were cultivated under strictly anaerobic conditions at $85^{\circ} \mathrm{C}$ in a rich growth medium (ASW-YT medium). ASW-YT medium was composed of a 1.25-fold dilution of artificial seawater ${ }^{9}$ (ASW), yeast extract $(5.0 \mathrm{~g} / 1)$, and elemental sulfur $(2 \mathrm{~g} / 1)(\mathrm{pH}$ 6.6). The cell suspension was then diluted 6-fold with distilled water and the fluores-

\footnotetext{
a) Author to whom correspondence should be addressed; electronic mail: oana@mech.t.u-tokyo.ac.jp
}

cent dye 4', 6-diamidino-2-phenylindole (DAPI) was added to visualize DNA and 2-mercaptoethanol (2-ME) was added as an antioxidant. The final concentration of each component was $0.13 \times \mathrm{ASW}-\mathrm{YT}, 0.5 \mu \mathrm{M}$ DAPI, and $4 \%(\mathrm{v} / \mathrm{v}) 2-\mathrm{ME}$. For the optical trapping, a CW Nd:YAG laser (1064 nm, Spectra Physics) operating between 180 and $1800 \mathrm{~mW}$ was introduced into a $100 \times$ oil-immersed objective and focused on the stage of an inverted fluorescence microscope (Nikon, TE-300). An auto-stage (Sigma Koki) was used to control the movement of a trapped object under the microscope. Fluorescence images were captured by a high sensitivity video camera (AP-Imager, Hamamatsu Photonics) and recorded on DV-video tapes. These experiments were conducted at a room temperature of $\sim 22{ }^{\circ} \mathrm{C}$.

Figure 1(a) shows an image of a KOD1 cell obtained by scanning electron microscopy (SEM) as in a previous work. ${ }^{10}$ The KOD1 cell measures $\sim 1 \mu \mathrm{m}$ in diameter. When a suspension of these cells was diluted with distilled water, most of the cells burst due to osmotic shock. Figures 1(b) and 1(c) show fluorescence images of cells before and after bursting. Since the fluorescent dye DAPI stains not only DNA but also cell membranes, whole KOD1 cells were visualized as shown in Fig. 1(b). In the burst cell, in Fig. 1(c), a snowman-like object was observed. It seemed to consist of a wreck of cell membrane (faint fluorescent object of $\sim 1 \mu \mathrm{m}$ in diameter) and a released genome DNA molecule (brighter fluorescent object of $\sim 2 \mu \mathrm{m}$ in diameter). To con-
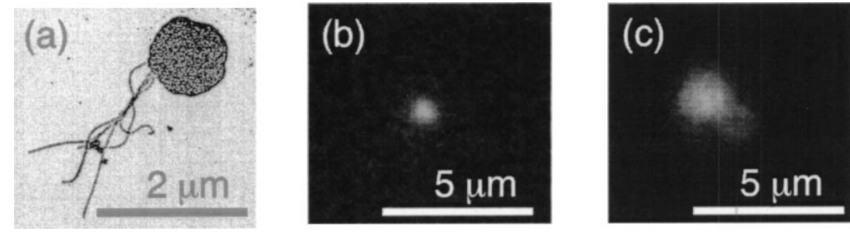

FIG. 1. (a) A SEM image of a KOD1 cell. (b) A fluorescence image of a KOD1 cell in ASW-YT medium visualized by DAPI. (c) A typical image of a burst KOD1 cell in dilute ASW-YT medium, where an empty cell membrane (lower right, faint smaller object) and the released genome DNA (upper left, bright bigger object) are visualized by DAPI 


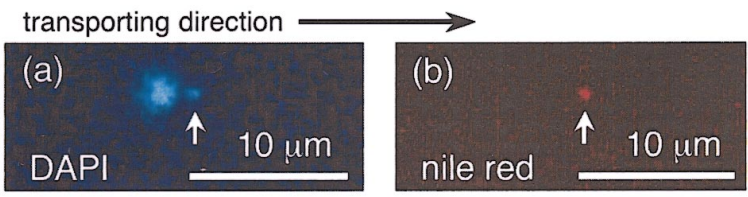

FIG. 2. (Color) Fluorescence images of a double-stained burst cell. (a) Image of a burst cell visualized by DAPI. (b) The same burst cell visualized by nile red. Arrows show the point trapped by optical tweezers.

firm this, a double-staining experiment was carried out. As an additional fluorescent dye, nile red (Invitrogen), which is a red fluorescent dye that is selective for neutral lipids, was added to $0.2 \mu \mathrm{M}$. Thus, DAPI stains DNA and cell membranes while nile red stains only lipids, i.e., cell membranes. Each fluorescence was observed by switching between emission filters.

Figure 2 shows images of a double-stained burst cell dragged by optical tweezers in diluted ASW-YT. When the laser focus approached the burst cell, the faint fluorescent part was attracted and immediately trapped. In the DAPI image in Fig. 2(a), the smaller fluorescent object is trapped and the connected larger object is trailing. On the other hand, in the nile red image in Fig. 2(b), the trapped smaller object is seen while the larger fluorescent object is invisible. These results suggest that the snowman-like object [Fig. 1(c)] is a cell membrane-bound released DNA. Since lipid molecules, which are major components of the cell membrane, have large permittivity ${ }^{11}$ and the trapping force of optical tweezers is due to the greater permittivity of the object compared to that of the surrounding solvent, the cell membrane can be trapped optically. When the cell membrane part was trapped, it seemed to shrink at the laser focus and the fluorescence intensity became brighter [compare Figs. 1(b) and 2(a)]. This phenomenon is due to the high permittivity of the cell membrane and the potential profile of the laser around the focused region, ${ }^{12,13}$ i.e., the cell membrane did not shrink but rather was deformed into a cylinder and aligned along the optical axis. Meanwhile, the released DNA part (larger fluorescent object) could not be trapped optically with an input as high as $1800 \mathrm{~mW}$. This difficulty in trapping the released DNA condensate might be due to its low packing density. In some previous studies, DNAs that were tightly packed by condensing reagents could be trapped optically. ${ }^{14,15}$ In one of these papers, DNA of $166 \mathrm{~kb}$ was highly packed in a particle on the order of submicrometer. ${ }^{14}$ On the other hand in our experiments, the released genome DNA exhibits somewhat swollen state. The successful transportation of the genome DNA in the present experiment is owing to the survival of the dense part in a giant genome DNA through the complex formation with the wreck of the cell membrane. This locally dense part may have some concern with specific regions on the genome, such as the replication origin, being attached rather tightly to the membrane in a living cell. To examine such possibility in connection with the genetic activity may be an important issue for future research.

As shown in Fig. 3, we carried out transportation of folded genome DNA into a high salt concentration to unwind the DNA. For this purpose, we constructed a chamber that consisted of cover slips and spacers (Fig. 3, top), as in a previous study by our group. ${ }^{7}$ The folded DNA was transported precisely by a combination of optical tweezers and an auto-stage. Before the burst cell was moved to the high-salt Downloaded 30 May 2007 to 130.54 .110 .22 . Redistribution subject
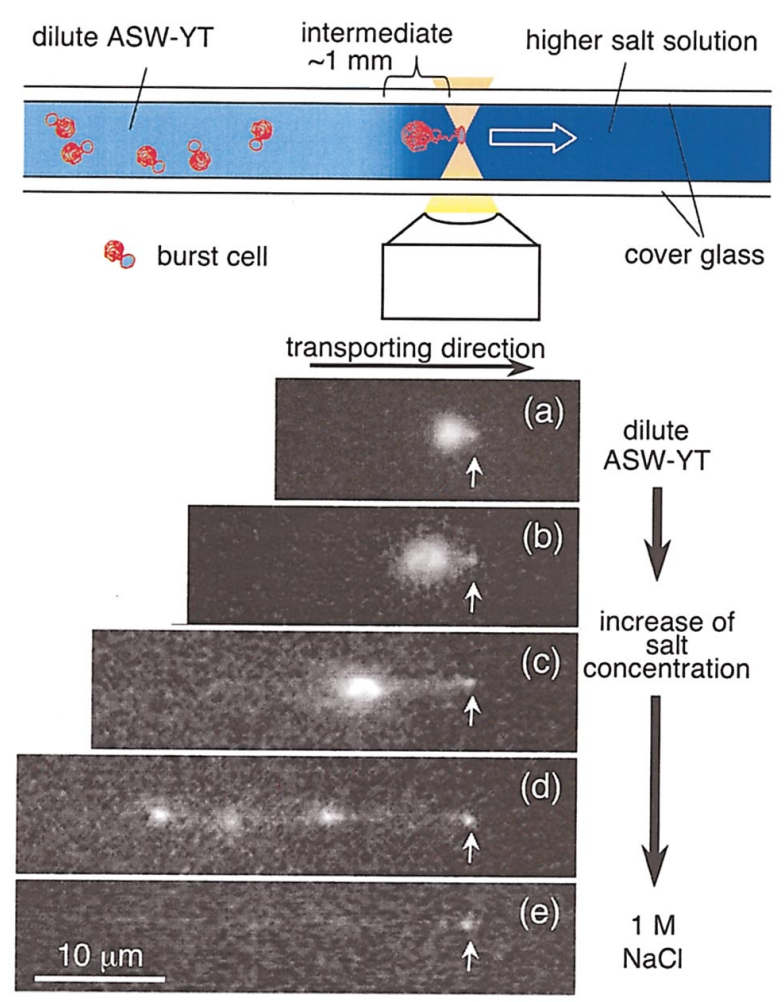

FIG. 3. (Color) Top: Schematic cross-sectional view of the experimental setup. In the dilute ASW-YT region, most of the cells are burst. The single genome DNA is trapped and transported to a higher salt region through an intermediate region by optical tweezers. The thickness of the solution was $\sim 200 \mu \mathrm{m}$. Bottom: Unfolding of a single KOD1 genome DNA. The folded DNA is transported into $1.0 \mathrm{M} \mathrm{NaCl}$ solution (right side) from the dilute ASW-YT (left side) through the intermediate region. White arrows indicate trapping points.

region, the burst cell was dragged in dilute ASW-YT medium. The burst cell was trapped tightly at the cell membrane and could be moved as fast as $200 \mu \mathrm{m} / \mathrm{s}$ with a laser input of $1800 \mathrm{~mW}$. During dragging, the folded part of the DNA maintained a spherical conformation and unfolding did not occur. Assuming that the dragged genome DNA is a hard sphere of $2 \mu \mathrm{m}$ in diameter and the viscosity of the solution is $1 \times 10^{-3} \mathrm{Ns} / \mathrm{m}^{2}$, the sphere feels $\sim 4 \mathrm{pN}$ when it is dragged at $200 \mu \mathrm{m} / \mathrm{s}$. This force $(4 \mathrm{pN})$ should be high enough to straighten DNA molecules in a random coil state, but is insufficient for stretching out DNA. ${ }^{16,17}$ Thus, this stability of the complex of DNA and histone-like proteins seems to be comparable to that of individual chromatin fibers. ${ }^{18-20}$ We then conducted transporting experiments from dilute ASW-YT to $1.0 \mathrm{M} \mathrm{NaCl}$ solution. First, in the dilute ASW-YT region, one of the burst cells was trapped and transported toward the high-salt region at $10 \mu \mathrm{m} / \mathrm{s}$ [Fig. 3(a)]. As the trapped cell began to enter the intermediate region, the folded DNA part started to swell and the dissociated part of the DNA was pulled out from the ball by the force of dragging [Figs. 3(b) and 3(c)]. As transporting continued, the distance between the trapping point and major folded part increased, since the DNA and histone-like proteins continued to dissociate. In addition, the large ball also began to show a trailing unfolded DNA [Fig. 3(c)]. The dragging force applied to the folded DNA was the driving force for pulling out and straightening the tethered part of DNA. The force acting at the connection between the tethered and ball parts during dragging at $10 \mu \mathrm{m} / \mathrm{s}$ can be estimated to be to AlP license or copyright, see http://apl.aip.org/apl/copyright.jsp 


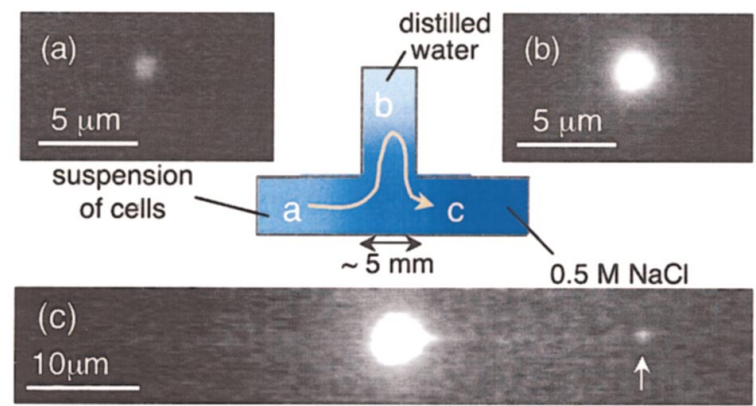

FIG. 4. (Color) Center: Schematic top view of the experimental field for the on-site manipulation of a single cell. Three different solutions (cell suspension, distilled water, $0.5 \mathrm{M} \mathrm{NaCl}$ ) are encountered and the trapped cell is exposed to each condition in turn. The arrow indicates the trajectory of the transported cell. (a) A KOD1 cell in the suspension before bursting. (b) The cell just after bursting by being transported into the distilled-water region. (c) Transported DNA toward $0.5 \mathrm{M} \mathrm{NaCl}$ solution. The white arrow indicates the trapping point.

sub-pico $\mathrm{N}$ and the tethered part is not stretched out. This strength is quite small compared to the force needed to dissociate histones from DNA in low concentration solutions of $\mathrm{NaCl}$ less than $150 \mathrm{mM} .^{18-20}$ Therefore, in our experiments, the irreversible dissociation of proteins, i.e., unfolding, was caused not by the dragging force but by the decrease in translational entropy gain due to the release of small counter ions. ${ }^{21}$ During the unfolding process, a partially folded conformation (the coexistence of a folded part and a random coil part in a single molecule) was observed [see Fig. 3(d)]. When the trapped genome DNA was moved completely into the high-salt region, the whole DNA became a random coil. In Fig. 3(e), the dragged DNA looks like a comet and the trailing end is outside of the observation area. In our experiments, the observation area on the monitor was at most $\sim 100 \mu \mathrm{m} \times 80 \mu \mathrm{m}$. In Fig. 3(e), the trailing part is very faint, since that part is in the random coil state and is affected by photo-bleaching. In other operations, when trapped genome DNA was transported to $0.5 \mathrm{M} \mathrm{NaCl}$ solution from dilute ASW-YT, the genome DNA also unfolded. We also carried out experiments in which burst cells were moved from dilute ASW-YT to low-salt conditions $(0.1 \mathrm{M} \mathrm{NaCl})$. As expected, a significant conformational change did not occur and the DNA maintained the same conformation as in dilute ASW-YT, a similar conformation shown in Fig. 3(a).

Next, we arranged three different solutions on a glass plate and carried out trial experiments on the on-site manipulation of a single whole-genome DNA. Figure 4 (center) shows a schematic drawing of the experimental field and the trajectory of a transported cell. First, a nonburst cell was trapped in the region in which cells were suspended. The trapped cell was then transported toward the bursting region, i.e., the distilled-water region (dissolved DAPI and 2-ME). Due to osmotic shock, the cell burst and the genome DNA rushed out from the cell [Fig. 4(b)]. DAPI immediately bound to the DNA and the fluorescence intensity became high. The burst cell was then transported to the unfolding region, i.e., high-salt region, and the genome DNA started to unfold. As shown in Fig. 4(c), dissociated DNA is pulled from the trailing ball part by dragging. Finally, when the genome DNA completely enters the high-salt region, it comes to be completely unfolded, as in Fig. 3(e).

In this study, we demonstrated that single whole-genome DNA could be recovered intact from a cell suspension under a microscope. In addition, the whole-genome DNA was manipulated using optical tweezers and its higher order structure could be changed by controlling the field conditions. The promising methodology demonstrated here may make it possible to recover an intact single whole-genome DNA from a cell and carry out further sequential investigations under a microscope. It is also expected that the long-standing dispute on the structure-activity relationship of genomic DNA, or chromatin, is clarified with the application of the present experimental methodology. Although it has been well known that the tightly packed region in chromatin, heterochromatin, is genetically silent and loosely packed, or partially unfolded, region, euchromatin, is active, the detailed mechanism to control the manner of DNA packing in relation to the genetic activity has not been unveiled yet. ${ }^{22}$

This work was supported in part by a Grant-in-Aid from the Ministry of Education, Science, Sports and Culture of Japan.

${ }^{1}$ For example, http://www.ncbi.nlm.nih.gov/Entrez/

${ }^{2}$ A. Ashkin, Proc. Natl. Acad. Sci. U.S.A. 94, 4853 (1997).

${ }^{3}$ Description of Thermococcus kodakaraensis sp. nov., a well-studied hyperthermophilic archaeon previously reported as Pyrococcus sp. KOD1. H. Atomi, T. Fukui, T. Kanai, M. Morikawa, and T. Imanaka, Archaea 1, 263 (2004)

${ }^{4}$ T. Imanaka and H. Atomi, The Chemical Record 2, 149 (2002).

${ }^{5}$ H. Higashibata, S. Fujiwara, M. Takagi, and T. Imanaka, Biochem. Biophys. Res. Commun. 258, 416 (1999).

${ }^{6}$ H. H. Ohlenbusch, B. M. Olivera, D. Tuan, and N. Davidson, J. Mol. Biol. 25, 299 (1967).

${ }^{7}$ Y. Yoshikawa, S-i. M. Nomura, T. Kanbe, and K. Yoshikawa, Chem. Phys. Lett. 330, 77 (2000).

${ }^{8}$ S. B. Zimmerman, J. Struct. Biol. 147, 146 (2004).

${ }^{9}$ F. T. Robb and A. R. Place, Archea: A Laboratory Manual - Thermophiles (Cold Spring Harbor Laboratory, New York, 1995).

${ }^{10}$ M. Morikawa, Y. Izawa, N. Rashid, T. Hoaki, and T. Imanaka, Appl. Environ. Microbiol. 60, 4559 (1994).

${ }^{11}$ R. Bar-Ziv, E. Moses, and P. Nelson, Biophys. J. 75, 294 (1998).

${ }^{12}$ S-i. M. Nomura, T. Harada, and K. Yoshikawa, Phys. Rev. Lett. 88, 093903 (2002).

${ }^{13}$ M. Ichikawa, Y. Matsuzawa, Y. Koyama, and K. Yoshikawa, Langmuir 19, 5444 (2003)

${ }^{14}$ Y. Matsuzawa, K. Hirano, K. Mori, S. Katsura, K. Yoshikawa, and A. Mizuno, J. Am. Chem. Soc. 121, 11581 (1999).

${ }^{15}$ C. L. Kuyper, G. P. Brewood, and D. T. Chiu, Nano Lett. 3, 1387 (2003).

${ }^{16}$ J. Zlatanova and S. H. Leuba, J. Muscle Res. Cell Motil. 23, 377 (2002).

${ }^{17}$ C. Bustamante, Z. Bryant, and S. B. Smith, Nature (London) 421, 423 (2003).

${ }^{18}$ Y. Cui and C. Bustamante, Proc. Natl. Acad. Sci. U.S.A. 97, 127 (2000).

${ }^{19}$ M. L. Bennink, S. H. Leuda, G. H. Leno, J. Zlatanova, B. G. de Grooth, and J. Greve, Nat. Struct. Biol. 8, 606 (2001).

${ }^{20}$ B. D. Brower-Toland, C. L. Smith, R. C. Yeh, J. T. Lis, C. L. Peterson, and M. D. Wang, Proc. Natl. Acad. Sci. U.S.A. 99, 1960 (2002).

${ }^{21}$ F. Oosawa, Polyelectrolytes (Dekker, New York, 1971).

${ }^{22}$ T. A. Brown, Genomes (BIOS Scientific, Oxford, 2002). 